\title{
A cluster mode-coupling approach to weak gelation in attractive colloids
}

\author{
${ }^{1,2}$ K. Kroy, ${ }^{2}$ M. E. Cates, and ${ }^{2}$ W. C. K. Poon \\ ${ }^{1}$ Hahn-Meitner Institut, Glienicker Str. 100, 14109 Berlin, Germany and \\ 2 School of Physics, The University of Edinburgh, Edinburgh EH9 3JZ, United Kingdom
}

\begin{abstract}
Mode-coupling theory (MCT) predicts arrest of colloids in terms of their volume fraction, and the range and depth of the interparticle attraction. We discuss how effective values of these parameters evolve under cluster aggregation. We argue that weak gelation in colloids can be idealized as a two-stage ergodicity breaking: first at short scales (approximated by the bare MCT) and then at larger scales (governed by MCT applied to clusters). The competition between arrest and phase separation is considered in relation to recent experiments. We predict a long-lived 'semi-ergodic' phase of mobile clusters, showing logarithmic relaxation close to the gel line.
\end{abstract}

PACS numbers:

Hard-sphere colloids with short-range attractions can undergo several types of arrest. At high densities they show two distinct glass transitions (repulsion-driven and attraction-driven), with a re-entrant dependence on attraction strength 1]. This scenario was first predicted by mode coupling theory (MCT) [2, 3, 4], and depends on both the attraction range $\delta$ (in units of particle diameter) and well-depth $\varepsilon$ (in units of $k_{B} T$ ). MCT is remarkably successful, at least for large volume fractions $\phi \gtrsim 0.4$.

At lower volume fractions, however, there is no comparable theoretical framework. Yet 'weak gelation', in which bonding is strong but not so strong as to be irreversible, can lead to nonergodic soft solids, of nonzero static elastic modulus, at volume fractions of just a few percent [5]. It might be argued that a finite modulus requires a percolating network of bonds whose lifetime exceeds that of the experiment. However this is simplistic: as shown by the case of repulsive glasses, a finite modulus can arise with no bonding at all. We argue here that the rigidity of weak gels arises not from bond percolation but from kinetic ergodicity breaking [6], just as it does in glass formation. This suggests that an MCT-like approach to weak gelation could be fruitful.

MCT takes its structural input from equilibrium liquid state theory; it cannot address states of arrest where this structure is strongly perturbed 7]. This matters relatively little at $\phi \gtrsim 0.4$, where each particle interacts with many others, and not much room is left for structural development upon a quench. But more severe consequences must be expected at low volume fractions where strongly nonuniform, ramified gels arise. Here the pathway to complete nonergodicity (starting from a homogenized fluid sample, say) must involve a nontrivial episode of structure formation, akin to irreversible cluster aggregation (ICA). Such kinetics certainly dominates for irreversible ("strong") gelation $\left(\varepsilon^{-1}=0\right.$ ), where particles aggregate on contact into clusters, with various kinetic universality classes [8]. Relative simplicity is restored at low $\phi$ thanks to the invariance of this aggregation process under coarse graining in the (ordered) limit $\delta, \varepsilon^{-1} \rightarrow 0$ and $\phi \rightarrow 0$. This scaling limit is controlled by an ICA 'fixed point', where details of the short-ranged attraction are irrelevant. The resulting fractal clusters grow indef- initely only if $\phi=0$; for $\phi>0$ they eventually form a percolating gel of locally ICA-like structure [9].

In this Letter, we explore how ICA might connect to weak gelation through a unified (albeit speculative) MCT-based scenario of colloidal arrest. We consider the changes in the system parameters $\left(\phi, \delta, \varepsilon^{-1}\right)$ upon coarse graining in the vicinity of the ICA fixed point. This leads to a schematic description of the suspension in terms of an effective theory for a dense liquid of 'renormalized particles' or coarse-grained clusters, for which recent simulations [10] seem to provide some direct evidence. Applying MCT to this theory, we obtain a new condition for arrest of the clusters, and identify the arrested state with the weak gel phase. If this condition is not met, one has instead a fluid of clusters (or 'cluster phase' [11]) that is ergodic at large scales. We allow for bond-breaking and reconnection at the scale of entire clusters, but not reconstruction at shorter scales. We return to this later, where we also address the potentially complex interplay between weak gelation and phase separation.

Within our scenario, weak colloidal gelation emerges as a double ergodicity breaking: once when the original suspension becomes kinetically unstable against aggregation, and a second time when the fluid of clusters arrests. We suppose that the criterion for the initial instability is given by bare MCT [6]. We assume $1 \ll \varepsilon<\infty$ : bonds can break with a small but finite probability. Initially this has almost no effect on the aggregation, which flows towards the ICA fixed point. But as clusters grow, the chances of fragmentation increase. The ICA fixed point is unstable and the sytem eventually flows away from it. The flow takes place in a parameter space comprising effective system variables $\left(\tilde{\phi}, \tilde{\delta}, \tilde{\varepsilon}^{-1}\right)$ (Fig. 1).

These coarse-grained parameters describe the density and interaction of aggregated clusters. We write:

$$
\begin{aligned}
& \tilde{\phi}=\phi N^{3 / d_{c}-1} \\
& \tilde{\delta}=\delta N^{-\nu} \\
& \tilde{\varepsilon}=\varepsilon-\chi \ln N-f(N)
\end{aligned}
$$

and call the combination of MCT with Eqs.(13) 'cluster mode-coupling theory' (CMCT). $N$ is the number of particles in a typical cluster, which is a parametric la- 
bel along flow trajectories, each of which is indexed by values of $\left(\phi, \delta, \varepsilon^{-1}\right)$ in the initial state after the quench. In Eqs.(12), evolution of the effective volume fraction of clusters (viewed as quasi-spherical, smooth objects) involves a scaling index $d_{c}$, and that of the effective relative range $\delta$ of their attraction another positive exponent $\nu$.

More subtle is the evolution of the bond energy $\varepsilon$ in Eq.(3). Since the ICA fixed point leads only to singlyconnected bonding, there is no power of $N$ multiplying the short-range attractive part of the bond energy. However, Eq.(3) accounts for bond breaking through a renormalization of the cluster-cluster attraction $\varepsilon$ by a breaking entropy, which we write as the logarithm of a breaking degeneracy $N^{\chi} \leq N$. This acknowledges that a ramified cluster falls apart when any of the $N^{\chi}$ bonds along its backbone is broken (with probability $\sim e^{-\varepsilon}$ ). Eq.(3) also includes a term $f(N)$ arising from additional interactions. For purely short range attractions this term should vanish, but it allows us to address also the case of a weak long range repulsion, such as has recently been shown to arise from poorly screened dissociated surface charges even for supposedly neutral colloids in organic solvents 12]. Depending on the strength and range of the repulsion, the resulting $f(N)$ may dominate the logarithmic term in Eq.(3) without having much effect on the equilibrium phase diagram [13].

Initially, all three exponents introduced in the CMCT equations are properties of the ICA fixed point (typically $d_{c} \approx 2, \nu \approx \chi \approx 1 / d_{c}$, in three dimensions [8]). However, they will start evolving once the flow moves away from it, as the internal structure of clusters evolves. An explicit treatment of this drift is not attempted here: we treat $d_{c}, \nu, \chi$ as negotiable constants.

The resulting $\mathrm{CMCT}$ flow within the renormalized parameter space $\left(\tilde{\phi}, \tilde{\delta}, \tilde{\varepsilon}^{-1}\right)$ is sketched in Fig. 1] The flow is bounded by the MCT transition surface in this space, which divides ergodic (upper left) from nonergodic regions. This surface 2, 3, 4] comprises a dome joined to a sheet. Below the dome lie attractive glasses and to the right of the sheet, repulsive ones. Note the presence of a seam $\tilde{\phi}_{c}$, on the transition surface, along the line where the sheet meets the dome. Consider a trajectory of increasing $N$ which rises up through the dome, left of the seam $\left(\phi<\tilde{\phi}_{c}\right)$. At this point, aggregation ceases, because the clusters' effective parameter values now correspond to an ergodic phase. A semi-ergodic fluid of finite clusters can be expected whenever the initial parameter values belong to such a trajectory. (This requires small $\delta$.) If a series of such cluster fluids is now created by increasing $\phi$ at fixed $\varepsilon$ (or vice versa), then the last of these to enter an ergodic phase will do so just beside the seam $\left(\tilde{\phi} \rightarrow \tilde{\phi}_{c}^{-}\right)$. Accordingly the first fully nonergodic phase formed has a trajectory that just meets this seam; beyond lies a repulsive glass, so that aggregation ceases. Unlike the cluster fluid, the resulting marginal gel has finite modulus - as do the non-marginal gels arising at larger $\phi$ or $\varepsilon$. This not because of percolation, which for reversible bonding is not relevant, but because the

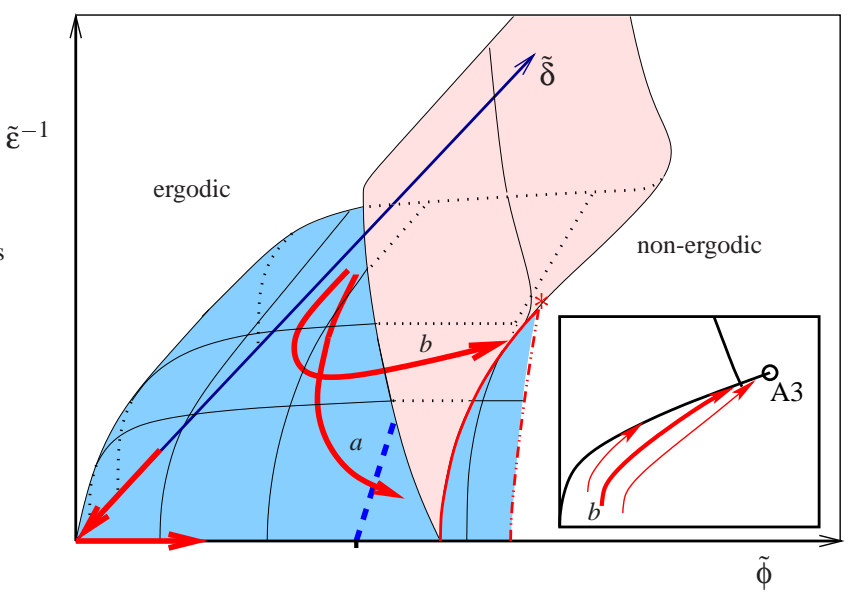

FIG. 1: Schematic parameter flows (arrows) near the ICA fixed point (at the origin, bottom left). Flow $(a)$ within the lower coordinate plane $\left(\varepsilon^{-1}=0\right)$ gives percolation of ICA clusters on passing through a non-equilibrium percolation line (heavy dashed). For $f(N) \geq 0$, trajectories starting from finite $\varepsilon^{-1}$ flow upwards to smaller $\tilde{\varepsilon}$ and towards larger $\tilde{\phi}$ and smaller $\tilde{\delta}$. Those that arrive on the domed dark surface result in semi-ergodic cluster phases. The marginal gel trajectory (b) ends at the seam where the repulsive (light surface) and attractive (dark surface) branches of the MCT transtion meet, close to a line of $A_{3}$ singularities (dash-dotted) or/and an $A_{4}$ singularity $(*)$. Inset: $\tilde{\phi}-\tilde{\varepsilon}^{-1}$-projection of trajectories leading to cluster or gel phase (thin) and marginal gel (b).

CMCT flow has led to a "cluster glass" which we identify with the weak gel phase. A typical phase diagram predicted by this approach is shown in Fig. 22

Note that for moderate $\tilde{\delta}$, the seam is not too far from the line of $A_{3}$ singularities, which is associated with a distinctive arrest scenario including a large exponent for the divergence of the structural relaxation time, and strongly stretched exponential or even logarithmic decay of the density correlations $[4,14,15]$. Marginal gels could thus be influenced by these singularities even at low volume fractions, although the details of this depend on the initial range $\delta$ and on the exponent $\nu$.

CMCT clearly offers nontrivial predictions for cluster phases, weak gels and relaxation anomalies. But it neglects two important pieces of physics which may complicate the experimental situation considerably. Firstly, we allowed clusters to dissociate by bond breaking, but neglected any internal restructuring within the clusters. By allowing these to coarsen into globules, this could cause both the cluster fluid and weak gel phases to have finite lifetime. Moreover, for $f(N)=0$ the breaking and rebonding of the $\mathcal{O}(N)$ dangling ends within a cluster may cause local restructuring even before the flow reaches the MCT surface. This issue is less important in the presence of a weak, long-range repulsion $f(N)>0$ (e.g. 12]), which, like bond-breaking, drives an upward flow in Fig. 1 In favorable cases this could dominate 


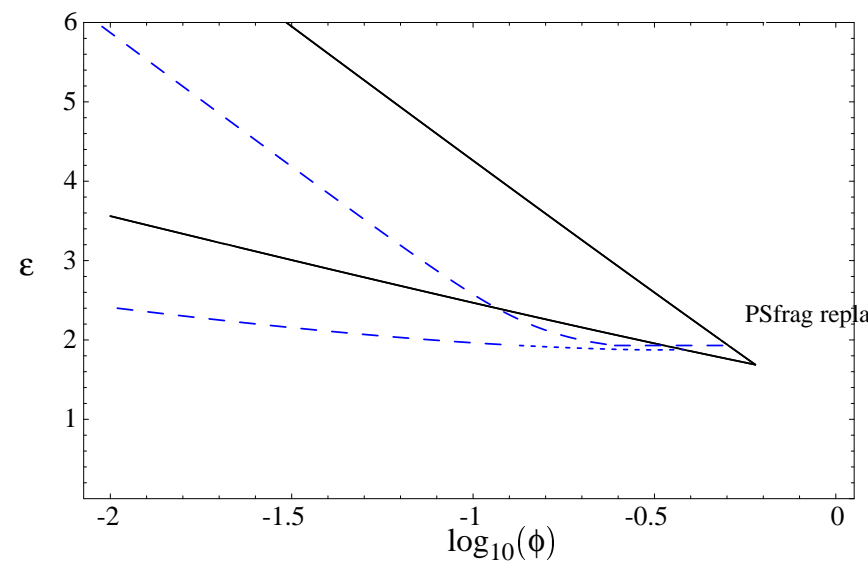

FIG. 2: Kinetic phase diagram predicted from CMCT with initial range of square-well attraction $\delta=0.1$. Exponents are chosen for definiteness as $d_{c}=2, \chi=\nu=1 / 2$ and $f(N) \equiv$ 0 . Bare MCT (in the low $-\phi$ approximation [6]) is used to estimate the onset of a clustering instability (lower solid line). The CMCT line divides cluster fluids from weak gels (upper solid line). Results allowing also for phase separation, using the indicative model of Fig. 3 give shifted phase boundaries (dashed lines). (The part of the equilibrium binodal above the AB tieline in Fig. 3 is also shown, dotted.)

the bond-breaking term at large $\varepsilon$, and allow the CMCT scenario to establish on a timescale during which local reconstruction remains negligible.

The second, closely related, omission from the CMCT predictions of Fig. 2 is the interplay of cluster formation with liquid-gas type phase separation [16]. To be confident of observing the CMCT line directly at small $\phi$, it must lie outside the binodal. When that condition is not satisfied, we expect results that depend on quench rates. For a very slow quench, the system first demixes locally and then coarsens, until the denser coexisting phase, whose properties continue to evolve, arrests by a CMCT-like mechanism (merging with bare MCT at high enough density). Slow quenches have recently been examined experimentally, and these ideas may account for a surprising "bead phase" seen at low $\phi$ [17].

For faster quench rates, local arrest can precede coarsening so that at small $\phi$, large fractal clusters will at first evolve, regardless of the underlying phase behavior. The resulting scenario is explored, within an indicative model, in Fig. 3] We follow Keller [18] in arguing that the densest phase capable of formation by demixing arises where the glass line intersects the binodal (point B). The nonergodicity line should depart from the CMCT prediction at this point, and track to lower densities along the tie line BA. This describes a locally demixed state in which only the denser (gel-like) B-component is nonergodic. At high $\phi$ the B-phase percolates giving a finite modulus gel; but at lower $\phi$ one expects a cluster fluid, now made of disconnected gel-like fragments. The 'gel line', separating states of finite modulus from those without,

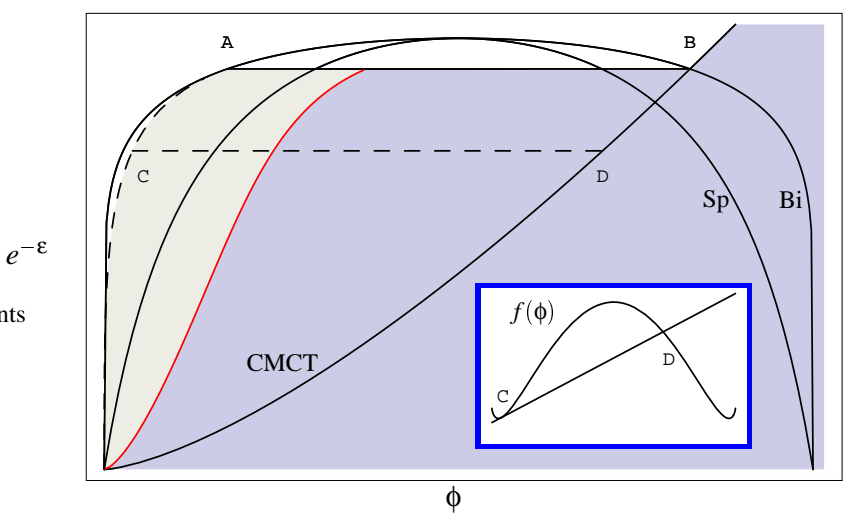

FIG. 3: Kinetic phase diagram, superimposed on the equilibrium phase diagram, for an indicative model. The equilibrium free energy density is that of a lattice gas, $f(\phi)=$ $\phi \ln \phi+\left(\phi_{m}-\phi\right) \ln \left(\phi_{m}-\phi\right)-\lambda \phi\left(\phi_{m}-\phi\right)$ with $\phi_{m}=0.64$ chosen as a maximum packing fraction. The second virial is matched to the colloidal system so that $\lambda(\varepsilon)=1 / 2-B_{2}(\varepsilon) v$ with $v$ the volume of a colloid; $B_{2}$ is that of a square well potential of range $\delta=0.1$. The CMCT line is found for the colloid system as in Fig. 2 equilibrium binodal (Bi) and spinodal (Sp) are marked. Inset: modified tangent construction on $f(\phi)[18$ in which states beyond D are deemed inaccessible. The gel line (with percolation threshold set at phase volume 0.3 of the arrested phase) bounds the darker shaded region. Between this and the modified binodal (dashed at left) lies the cluster fluid phase (lighter shading).

corresponds to the geometric percolation threshold of the arrested phase. Deeper quenches should behave similarly, with local coexistence between a dilute fluid $\mathrm{C}$ and an arrested state D whose density lies on the CMCT line. This is governed by a modified common tangent [18] in which inaccessible densities (beyond point D) are prohibited (Fig. 31 inset) shifting the binodals inward. Along the tieline $\mathrm{CD}$, the gel line again corresponds to percolation of the arrested phase. At still deeper quenches the CMCT line lies within a (renormalized) spinodal curve; this local instability complicates matters further, and is ignored in the indicative model of Fig. [3 But a transition from percolating gel to a cluster phase of gel fragments should remain. Finally, at infinite $\varepsilon$ the CMCT line, the gel line, the equilibrium spinodal, and the binodal all meet the ICA fixed point at the origin. Notably, this scenario allows the 'marginal gel' behavior to extend inside the gel phase: indeed a range of compositions, including the $\mathrm{AB}$ tieline and a region below it, now have local phase separation involving a marginal gel (Fig. 3).

We now confront the implications of our work with experimental data. The most direct consequence of our hypothesis of a double ergodicity breaking, a fractal gel structure, is a very common observation. It has inspired successful phenomenological gel models, e.g. 19, 20, 21], for which CMCT could provide a more rational (albeit still speculative) basis. Experiments 22] demonstrate that even for moderate attractions the assumed closeness 
to the ICA limit is well justified at low volume fractions in some cases, while in others the measured fractal dimension lies higher than for the appropriate ICA model 23. The latter could well be a consequence of a partial phase separation followed by arrest, as discussed above.

Secondly, CMCT predicts dynamic anomalies along and near much of the gel line (including the part that lies along $\mathrm{AB}$ tieline of Fig. B). In this context, the observation in Ref. 11] of a large exponent (5.5 \pm 1 at $\phi \approx 0.1)$ for the divergence of the terminal time, and of slow, possibly logarithmic, structural relaxation close to the gel line at low $\phi$ are both suggestive of CMCT.

A final comparison involves the nonequilibrium phase diagram, where a striking feature at low concentrations (e.g. 11, 21, 24]) is indeed the existence of a phase of large mobile aggregates, lying between the ordinary fluid phase and the gel. In fact, the phase diagrams in Fig. 5 of Ref. 11] and Fig. 2 of Ref. 25] are very similar to our Figs. 2] 3] respectively. As mentioned above, an interplay of CMCT with phase separation is expected for most colloidal parameters, including those of Ref. 11]. Fig. 2 therefore shows also the phase boundaries adjusted to allow for phase separation according to the indicative model of Fig. 3 In this representation the cluster/gel boundary remains nearly straight, although shifted downward somewhat. The results remain qualitatively consistent with Ref. 11]; given the indicative character of the model, this is encouraging. Since the system is among those for which a long range Coulomb repulsion might be present [12], it is possible that $f(N)>0$. Qualitatively, this would move the calculated gel line upward, stabilizing the cluster phase.

In summary, we have proposed a new framework to describe weak gelation based on mode-coupling theory applied to clusters (CMCT). This certainly contains speculative elements but gives nontrivial predictions, several of which compare well to existing experimental data. Underlying our picture of a double ergodicity breaking is the premise of a time scale separation. Our "cluster glass" view of weak gels is a meaningful concept on time scales longer than the time for cluster formation, but short compared to typical times over which a cluster reconstructs as it evolves towards equilibrium. This condition is more easily satisfied in systems where a repulsion between clusters builds up as they grow (e.g. weakly charged particles), limiting their final size. When the size is limited by bond-breaking, the required time scale separation is less likely. But even if the resulting weak gels are not true examples of broken ergodicity and are only temporary structures, CMCT may offer valuable insights into their behavior. Further experimentally relevant consequences of the scheme will be the subject of future work.

We thank Mark Haw, Daan Frenkel, Matthias Fuchs, Helen Sedgwick, Matthias Sperl and Dave Weitz for valuable discussions, and the EC for financial support.
[1] K. N. Pham, A. M. Puertas, J. Bergenholtz, S. U. Egelhaaf, A. Moussaid, P. N. Pusey, A. B. Schofield, M. E. Cates, M. Fuchs, and W. C. K. Poon, Science 296, 104 (2002).

[2] J. Bergenholtz and M. Fuchs, Phys. Rev. E 59, 5706 (1999).

[3] L. Fabbian, W. Götze, F. Sciortino, P. Tartaglia, and F. Thiery, Phys. Rev. E 59, R1347 (1999).

[4] K. Dawson, G. Foffi, M. Fuchs, W. Götze, F. Sciortino, M. Sperl, P. Tartaglia, T. Voigtmann, and E. Zaccarelli, Phys. Rev. E 63, 011401 (2001).

[5] W. C. K. Poon and M. D. Haw, Adv. Colloid Interface Sci. 73, 71 (1997).

[6] J. Bergenholtz, M. Fuchs, and T. Voigtmann, J. Phys.: Condens. Matter 12, 6575 (2000).

[7] It also neglects the residual dynamics still present in the arrested state, such as activated processes and aging [J.P. Bouchaud, L. Culiandolo, J. Kurchan, and M. Mezard, Physica A 226, 243 (1996)], and overestimates the tendency to arrest.

[8] T. Vicsek, Fractal Growth Phenomena (World Scientific, Singapore, 1992), 2nd ed.

[9] A. Hasmy and R. Jullien, Phys. Rev. E 53, 1789 (1996).

[10] E. Del Gado, A. Fierro, L. de Arcangelis, and A. Coniglio, Europhys. Lett. 63, 1 (2003).

[11] P. N. Segrè, V. Prasad, A. B. Schofield, and D. A. Weitz, Phys. Rev. Lett. 86, 6042 (2001).

[12] A. Yethiraj and A. van Blaaderen, Nature 421, 513 (2003).

[13] It slightly distorts the phase diagram (e.g. it shifts the repulsive glass transition to slightly smaller volume fractions), and may turn the spinodal into an instability towards micro-phase separation. These effects are not crucial to the present discussion.

[14] W. Götze and M. Sperl, Phys. Rev. E 66, 011405 (2002).

[15] F. Sciortino, P. Tartaglia, and E. Zaccarelli, condmat/0304192.

[16] M. A. Miller and D. Frenkel, Phys. Rev. Lett. 90, 135702 (2003).

[17] H. Sedgwick, K. Kroy, A. Salonen, M. B. Robertson, S. Egelhaaf, and W. C. K. Poon, cond-mat/0309616.

[18] F. C. Frank, A. Keller, Polym. Commun. 29, 186 (1988).

[19] W.-H. Shih, W. Y. Shih, S.-I. Kim, J. Liu, and I. A. Aksay, Phys. Rev. A 42, 4772 (1990).

[20] A. H. Krall and D. A. Weitz, Phys. Rev. Lett. 80, 778 (1998).

[21] V. Trappe and D. A. Weitz, Phys. Rev. Lett. 85, 449 (2000).

[22] A. D. Dinsmore and D. A. Weitz, J. Phys.: Condens. Matter 14, 7581 (2002).

[23] L. F. Rojas, R. Vavrin, C. Urban, J. Kohlbrecher, A. Stradner, F. Scheffold, and P. Schurtenberger, Faraday Discuss. 123, 385 (2003).

[24] W. C. K. Poon, L. Starrs, S. P. Meeker, A. Moussaïd, R. M. L. Evans, P. N. Pusey, and M. M. Robins, Faraday Discuss. 112, 143 (1999).

[25] H. Verduin and J. K. G. Dhont, J. Colloid and Interface Sci. 172, 425 (1995). 